\title{
A Modular Approach to Real-Time Cork Classification Using Image Processing
}

\author{
José Luis Lima \\ IPB - Instituto Politécnico de Bragança, \\ Escola Superior de Tecnologia e de Gestão. \\ Campus de Santa Apolónia, Apartado 1038 \\ 5301-854 Bragança Portugal \\ jllima@ipb.pt
}

\author{
Paulo Gomes Costa \\ FEUP - Universidade do Porto, \\ Faculdade de Engenharia. \\ Rua Dr. Roberto Frias, s/n, \\ 4200-465 Porto Portugal \\ paco@fe.up.pt
}

\begin{abstract}
This paper's main purpose is to present an automatic vision assortment system for cork classification. Cork is a natural material that is used to seal wine bottles due to its reliability and its chemical and mechanic properties. A modular approach isolates the hard real-time sub problems. It includes some custom developed hardware and software. The developed and implemented system acquires images using a linear CCD camera, transmits that image over an Ethernet based network and processes that image to extract quantitative elements. This system can analyse and distinguish defects present on the surface of a cork-stopper and it is also possible to adapt it to other kinds of problems. The size, form and position of the defects are analysed and the stability of repeated measures is observed. This result allows us to validate the presented methodology.
\end{abstract}

\section{Introduction}

Cork-stoppers are used to seal wine bottles because, unlike man-made material, they avoid leakage even in presence of irregularities in the bottle neck. They show an excellent chemical inertia and avoid any reaction with the wine that changes its taste. Their best characteristic is the ability to allow gaseous exchange with the outside atmosphere. This is an important step that makes it possible to improve the wine quality as time goes by [1].

An artificial vision system capable of cork classification is an important step to the homogenization of cork classification parameters. In fact, some cork manufacturers do this classification manually and there are no criteria to selection. Furthermore, the human tiredness influences the decision. The selection can vary from person to person or time to time.

So, each cork-stopper class is not uniquely defined, therefore a machine criterion can help in standardization.

The quality of a cork-stopper is decided by a function of several features, defects and the presence of insects [1]. The latter are not inspected because they typically appear on the top or bottom of the cork-stopper and the developed system only acquires the cylinder surface of the cork-stopper only. Furthermore, insects were a problem some years ago due to the bad storage conditions but, nowadays, this problem is partially solved.

The cork defects appear in the form of cracks, random-shaped holes, and others. Cork-stoppers are grouped into eight classes according to the degree of defects on its surface.

Modular acquisition and processing systems can improve the versatility of the manufacturing process. The presented system tries to accomplish these goals. This modular system is divided into acquisition and processing subsystems. The first one is a hard real-time system and the second one is a soft real-time system. Both are connected through an Ethernet based network that allows both subsystems to be separated.

The linear CCD camera and the signal conditioning system acquire and send the grey image to the computer through the network. This is a hard real time task performed by a microcontroller.

In the implemented software, cork images are acquired and processed but other kind of images can be used to test and validate image processing algorithms. These algorithms, such as morphologic manipulation, convolution, binarization and clustering are presented and tested using acquired and virtual images.

The real goal is to obtain an automatic classification system that allows a faster and right decision about the cork class. The modular approach can also lead to easier deployment on the field.

The paper is organized as follows: initially, Section 2 describes the mechanical and hardware subsystem. Section 3 describes the main image processing algorithms and Section 4 describes the classification methods and presents an example. Section 5 describes the real-time requirements of the system. Finally, Section 6 rounds up the paper with conclusions and presents the future work.

\section{General system description}

The developed system comprises two main subsystems: the mechanical subsystem which is responsible for the handling of cork-stoppers and the hardware/software subsystem which is responsible for acquiring, processing images and classification tasks. 


\subsection{Mechanical subsystem}

The mechanical system that appears as an assembly line is made up of three main parts: the feeder system, the inspection platform and the binning selection mechanism (with eight different outlets) as illustrated in Figure 1.

The feeder system lines up cork-stoppers in the inspection platform. There, a set of three rollers, with a uniform rotation movement, assures the rotation of the cork-stopper in front of the camera as shown in Figure 2.

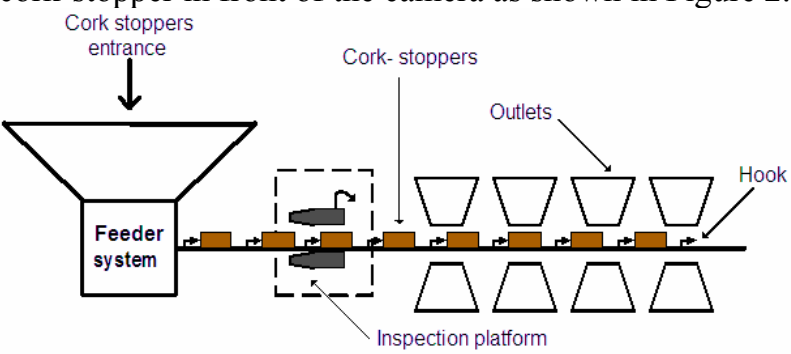

Figure 1. Mechanical subsystem.

The cork-stopper inside the inspection platform performs two movements simultaneous: one of translation, from the feeder system to the outlets, and a rotational movement produced by the rollers.

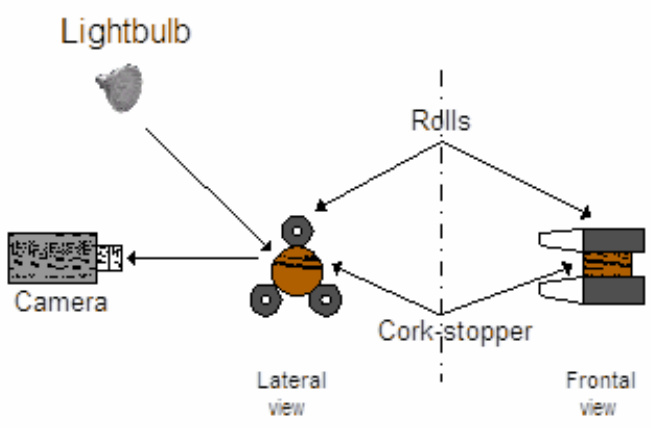

Figure 2. Lateral and frontal view of inspection platform.

Figure 3 presents a view of the developed system installed on real working environment.

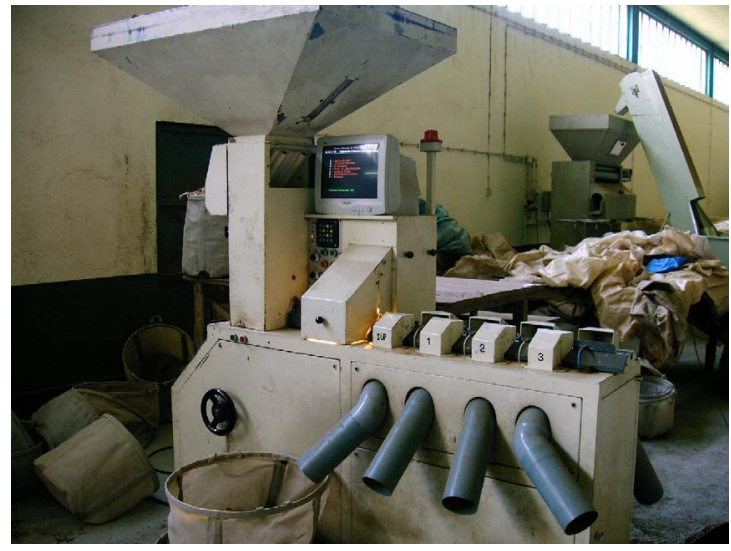

Figure 3. Mechanical view of the developed system.
The spin movement of the cork-stoppers allows the linear camera to acquire the cylinder surface completely like a flattened image. In this system, top and bottom are not processed due to reasons described in Section. 1.

\subsection{Hardware subsystem}

The developed electronic system is composed by several components. The global hardware architecture is described in Diagram 1.

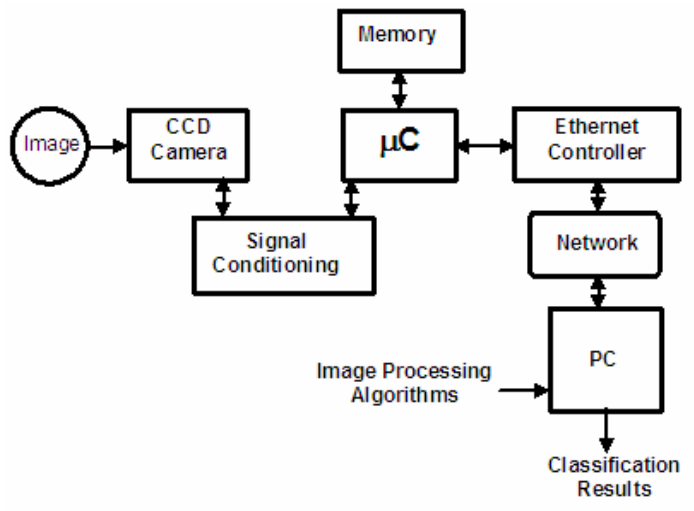

Diagram 1. Hardware architecture.

The main sensor is a CCD camera, which captures the cork image. The analogue electrical signal is conditioned and digitized by an A/D converter. After that, a fast microcontroller [2] sends each cork image through an Ethernet based network, line by line. The microcontroller is also used to excite and synchronize the CCD. The camera operates with a linear CCD [3] that acquires the rotational image with 110 lines and sets up a complete flat image. Lines are formed by 256 pixels which are scanned through clock synchronization. Each pixel (analog voltage) exits in serial form, as shown in Figure 4 , and is subsequently converted by an 8 bit flash A/D converter. Some initial and final pixels -dummy pixels- carry no light information. They are used to help in setting the voltage reference and noise minimization.

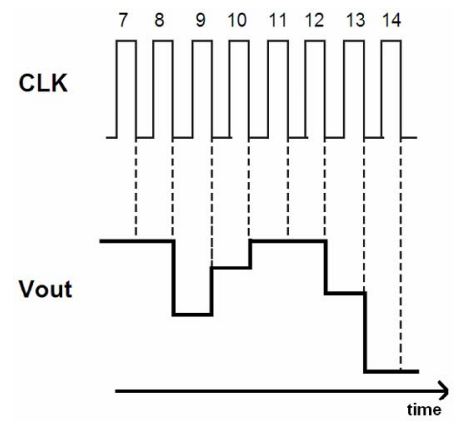

Figure 4. Pixels synchronization.

The real-time task is done by the microcontroller. The microcontroller has to scan pixels from the CCD, convert them and store each one in just $1 \mu$ s. This 
operation must be carried out until the end of the line. Here, the critical time requirements are all important.

The microcontroller stores the digital values for each line and sends them to the $10 \mathrm{Mbit} / \mathrm{s}$ Ethernet controller [4]. After that, it must be ready for the next line. The UDP protocol over a dedicated network is used in order to reduce the delay of travel time as a real-time requirement. The UDP protocol is very useful in realtime requirements due to its simplicity. There is no guarantee that transmission was complete, but in practice and with a dedicated network all the packets are transmitted correctly. This can be verified because all lines are tagged with an incremental number (line number).

It takes about $280 \mu$ s to obtain a line (real and dummy pixels) with a pixel scan frequency of $1 \mathrm{MHz}(1 \mu \mathrm{s}$ each pixel) and about $1.33 \mathrm{~ms}$ (256 bytes) to transmit each line which means a $1.5 \mathrm{Mbit} / \mathrm{s}$ of real transmission speed.

This way, it allows us to separate the acquisition system and the processing system. Having a distributed system connected by Ethernet makes it possible to have both systems distant from one another. And, the second one (a personal computer), as an independent system connected through network, is able to have any operative system and does not need a hard real-time OS because this subsystem has only soft real-time constraints (it only needs to be fast enough to process the image and to make the decision).

The final image is made up of 110 lines. Each has 256 pixels with 8 bits grey intensity. Real and acquired images are shown in Figure 5.

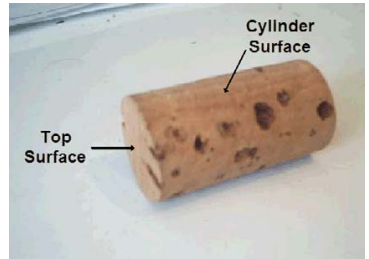

(a)

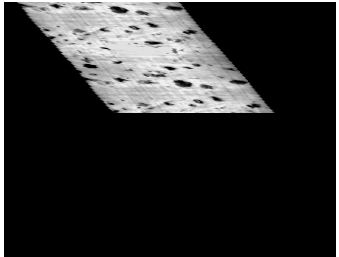

(b)
Figure 5. Cork-stopper image: (a) Real cork-stopper photo; (b) cork-stopper image acquired by a grey camera.

The illumination must be very carefully set up. It must be stable and include no $50 / 60 \mathrm{~Hz}$ ripple. A DC/DC controller converter was developed in order to guarantee these topics. The voltage controller gives an extra degree freedom to the light control adjacent to the iris lens control. To minimize the light intensity changes, an closed cabin accommodates the CCD camera. The stable light inside this cabin can be controlled by software.

\section{Image processing}

Well-known image processing algorithms such as the morphological manipulation; convolution; binarization and clustering are applied to the problem.

\subsection{Cork-stopper boundary}

The first processing algorithms find the localization of the cork-stopper image included in the original and acquired image. A skew algorithm is applied in order to obtain a square cork-stopper surface image as presented in Figure 6.

These processing algorithms try to find the horizontal and vertical limits. The horizontal limit can easily be found because it can be assumed that the cork-stopper image is inside a rectangle.

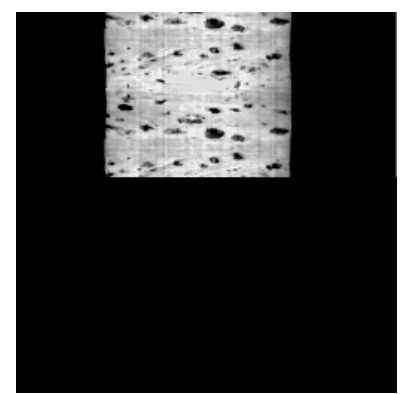

Figure 6. Cork-stopper image after skew algorithm.

One also needs to check whether the intensities of the first and the last columns are above some threshold or not. Intensity for one line is shown in Figure 7 such as the threshold level.

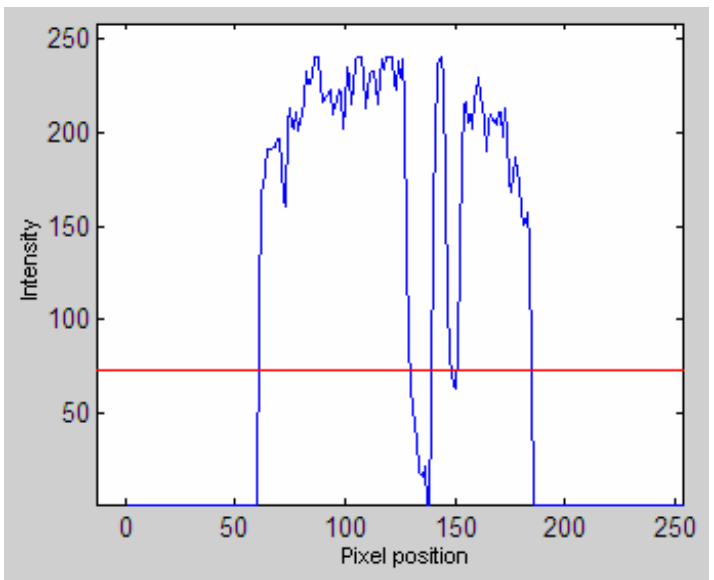

Figure 7. Line intensity.

For a statistical study, 256 samples of the same cork-stopper were measured and a histogram, illustrated in Figure 8, was made. The horizontal axis is the length in pixels and the vertical one is the number of measures. 
This histogram shows a good stability of measures with an average of 125.1 and a standard deviation of 0.55 .

A varying distance between the camera and the cork-stopper modifies its perceived length. In industrial systems, the camera is fixed and the cork-stoppers go forward helped by a hook. This way, distance is correctly kept. Besides, the cork-stopper length is a very important setting to manufacturers and it must be included in classification.

The cork-stopper length parameter can be used to calibrate the system if an inaccurate distance was set for the camera. By using a known object, calibration can be done.

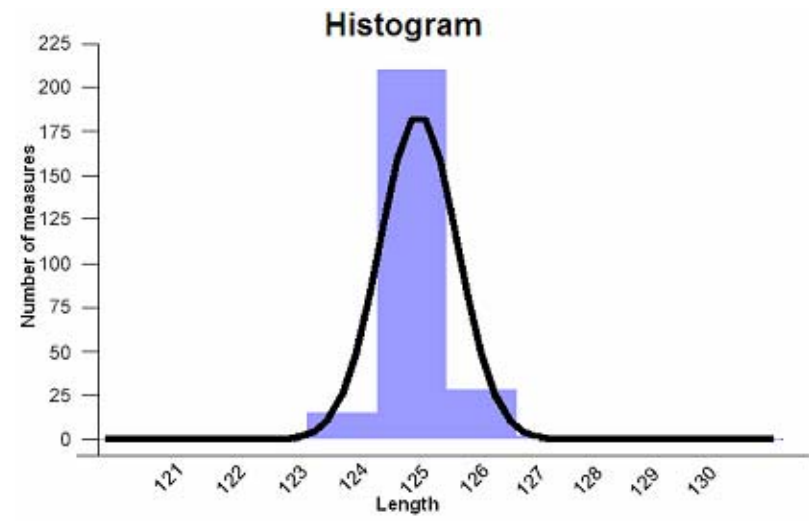

Figure 8. Histogram of cork-stopper length in pixels.

On the other hand, the vertical limit is more convoluted. In fact, the angular speed of the cork-stopper is not exactly constant over time. This problem arises from the sliding between cork surfaces and rollers that transmit movement to the cork-stopper. To solve this problem, the cork-stopper surface must be acquired more than once. Therefore, one has the guarantee that all the area is fully obtained. A correlative measure, $k$, between the three first lines and all the other lines, can be found. The minimum value of the measure of all lines gives the last line of the image. The formula for $k$ where $p_{i, j}$ is the pixel value, of the $i$ line and $j$ column is:

$$
k(\text { line })=\sum_{i=1}^{A} \sum_{j=0}^{B}\left|p_{i, j}-p_{i+l i n e, j}\right|
$$

Where $B$ is the image width and $A$ the number of lines to compare $(\mathrm{B}=255$ and $A=3)$.

The final result is shown in Figure 9 where the cork-stopper limits are automatically extracted from the acquired image.

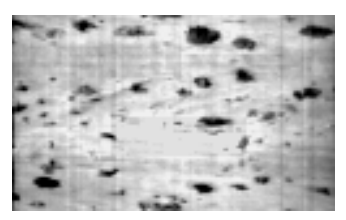

Figure 9. Cork-stopper image acquired and physically circumscribed.

The number line/cork is now known as the needed lines to represent the cork-stopper. If it was a closed-loop system, this number would be essential to feedback the angular speed.

This processing allows the system to work without an encoder attached to the cork rotation surfaces.

The defect measures should be done in millimetres. The pixel to millimetre transformation can be found by resorting to optics theory. Horizontally, the size of projected image, $H_{i m}$, in CCD can be found by:

$$
H_{i m}=\frac{H_{o b j} \cdot D_{f}}{D_{o b j}-D_{f}}
$$

where $H_{o b j}$ is the size of object, $D_{f}$ the lens focal distance and $D_{o b j}$ the distance from object to lens. This is possible because the barrel distortion can be ignored due to the quality of the used lens [7]. Vertically, object height in millimetres can be calculated from object in pixels by:

$$
O b j_{m m}=k_{2} \cdot O b j_{P I X}
$$

where

$$
k_{2}=\frac{\pi \cdot d}{\text { line } / \text { cork }}
$$

In this formula, $d$ is the cork-stopper diameter and line/cork is the number of acquired lines for a complete cork-stopper, as previously mentioned.

Of course, the measures in millimetres are more stable and invariable with rotation speed and camera distance than in pixels. To test it, the same cork-stopper was acquired with two different speeds. The defects were measured for both situations. The variation of defects in pixels and millimetres for both acquisitions are presented in Table 1. Longitudinal and transversal cracks are shown in Figure 10. 
Table 1. Pixels and millimetres variation with angular speed rotation.

\begin{tabular}{|c|c|c|}
\hline Defects & $\begin{array}{c}\text { Pixels } \\
\text { variation (\%) }\end{array}$ & $\begin{array}{c}\text { Millimetres } \\
\text { variation (\%) }\end{array}$ \\
\hline Area detected & $55.70 \%$ & $0.10 \%$ \\
\hline $\begin{array}{c}\text { Maximum Area } \\
\text { detected }\end{array}$ & $46.50 \%$ & $-5.80 \%$ \\
\hline $\begin{array}{c}\text { Maximum } \\
\text { longitudinal } \\
\text { crack }\end{array}$ & $0.00 \%$ & $0.00 \%$ \\
\hline $\begin{array}{c}\text { Maximum } \\
\text { transversal } \\
\text { crack }\end{array}$ & $55.00 \%$ & $-0.40 \%$ \\
\hline
\end{tabular}

As expected, the longitudinal defects are not affected by the speed rotation although they change with camera distance. But defects areas are strongly affected by the rotation speed leading to a higher transversal defects variation. This software layer tries to keep the measures stable.

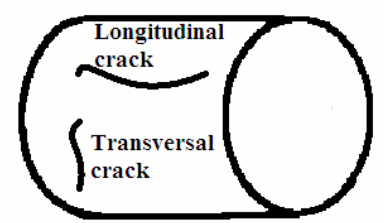

Figure 10. Longitudinal and transversal cracks.

\subsection{Image enhancement}

A second processing algorithm, histogram equalization or contrast enhancement, is applied. Not only to allow a better image visualization but also an improvement of the robustness of some algorithms, under changes in the light intensity.

The contrast enhancement can be applied to counterbalance an acquired image with low contrast. The algorithm tries to expand the histogram so that it occupies the range of all possible values: the minimum and the maximum bright found in the original image are converted to the minimum and maximum possible values for each pixel (0 and 255). In fact, some "salt and pepper" noise present in the acquisition phase prevents a direct application of the algorithm. The corrupted pixels are either set to the maximum value (which looks like snow) or to the minimum value (which looks like black points). A way to reduce this noise is to use a median filter. In this application, that was not necessary since there was no "salt and pepper" noise. The function associated with a contrast enhancement can be displayed as a graph for each input-output value as shown in Figure 11 .

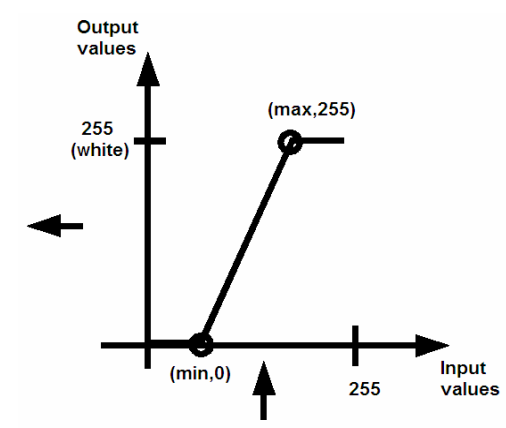

Figure 11. Transfer function of contrast enhancement.

For the input image shown in Figure 12 (a), a final result (c), histograms (b) and (d) and the contrast enhancement are displayed.

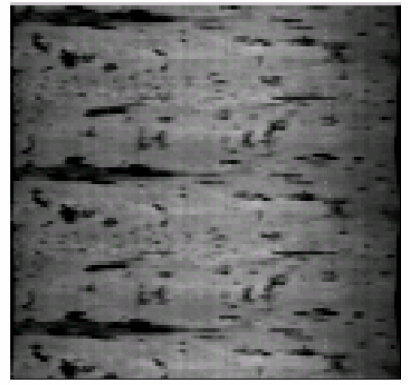

(a)

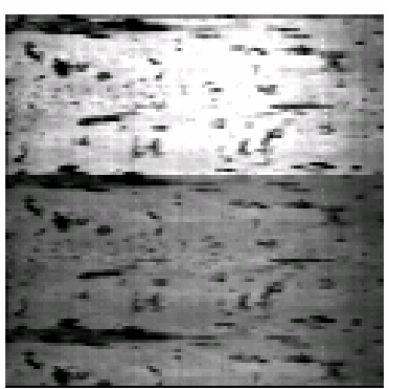

(c)

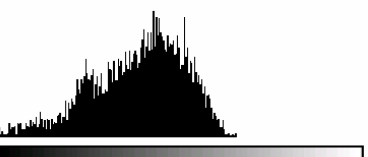

(b)

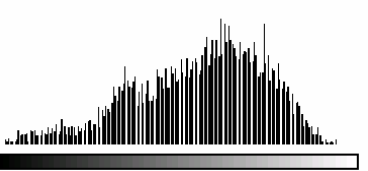

(d)
Figure 12. Images to equalize histogram: (a) original acquired image; (b) histogram of (a); (c) contrast enhancement of (a); (d) histogram of (c).

The final image (Figure 12-c) is only processed in the first cork-stopper region to minimize the processing time. So, only the needed area is enhanced.

\subsection{Edge detection}

The main task of the processing is to extract features of the cork-stopper surface like the location, area and type defects. An edge detection algorithm is the base of all classification tasks. Before classification, other filters are applied to minimize the effects from noise found during acquisition.

The third algorithm is a threshold operation to define the image defects. Some errors are introduced in the 
neighbourhood of the defects by this operation and must be dealt with. In practice, the longitudinal cracks and the extremity defects must be very carefully processed because they are critical for some cork properties.

Before classifying the longitudinal cracks, a convolution is applied so that the best results can be obtained.

Basically, the convolution is a window $H$ that is scanned across the original image $a$. The output pixel value is the weighted sum of the pixels within the window, where the weights are the values of the filter assigned to each pixel of the window itself. The window $H$ with its weights is called the convolution kernel. If the filter $H[i, j]$ is zero outside the rectangular window ( $\mathrm{i}=0,1, \ldots \mathrm{I}-1 ; \mathrm{j}=0,1, \ldots \mathrm{J}-1)$, the convolution can be written as the finite sum presented in (5)

$$
\begin{aligned}
& Y(m, n)=a(m, n) \otimes H(m, n)= \\
& =\sum_{i=0}^{I-1} \sum_{j=0}^{J-1} H(i, j) \cdot a(m-i, n-j)
\end{aligned}
$$

where $Y$ is the resulting image and $H$ is the convolution kernel (to vertical edge enhancement), given by (6) which gets very good results.

$$
H=\left[\begin{array}{ccc}
0 & 1 & 0 \\
0 & 1 & 0 \\
0 & -1 & 0
\end{array}\right]
$$

To exemplify it, the acquired image and all the detected defects are shown in Figure 13. The maximum length of the longitudinal crack is 15.35 millimetres.

The image convolution with the line mask (6) and all the detected defects are shown in Figure 14. The maximum length of the longitudinal crack is now 44.27 millimetres, just like the cork-stopper dimension.

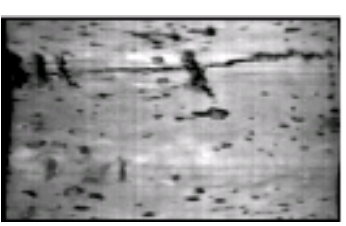

(a)

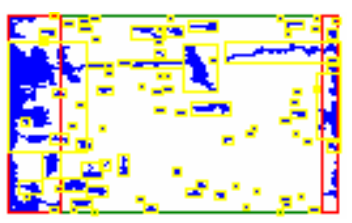

(b)
Figure 13. (a) Acquired image; (b) Black and white labelling.
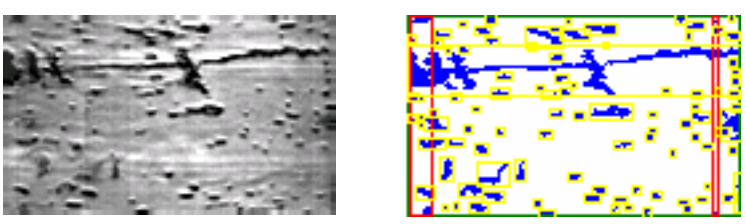

Figure 14. (a) Acquired image with convolution; (b) Black and white labelling.

To eliminate small defects and some noise introduced during the acquisition process and to calculate the value of the total defects area, an open nonlinear filter algorithm (erode followed by dilate) was tested with good results, as shown in Figure 15.

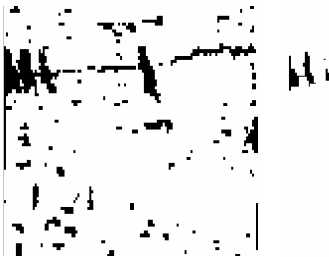

(a)

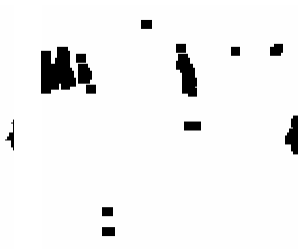

(c)
Figure 15. (a) Black and white image; (b) Eroded image; (c) Opened image.

A statistical study was made so that the accuracy of the measures of the areas could be verified.

The final result (in millimetres) of total area for 256 acquired images for the same real cork-stopper is shown by a histogram in Figure 16. The average is $333.40 \mathrm{~mm}^{2}$, and the standard deviation is $6.83 \mathrm{~mm}^{2}(2.65 \%)$. This results show the stability of the system.

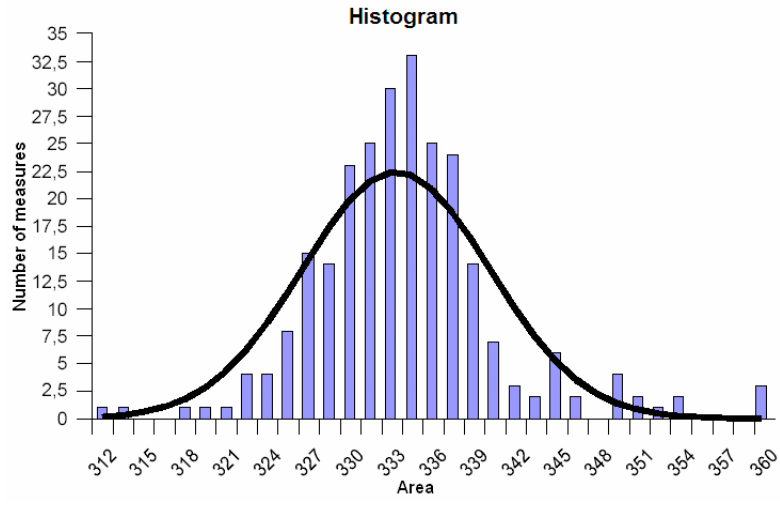

Figure 16. Histogram of total area defects.

\section{Classification}

The class decision of cork quality by human experts is established according to very complicated rules and each person can have his own. 
Cork-stoppers are grouped into eight classes according to the degree of defects on the cork-stopper surface.

There must be a configurable system because each cork manufacturer imposes his own "rules". Moreover, the worst class is cheaper and less profitable. So, cork manufacturers try to shift worst classes the best they can.

The classification process is to pick up a class for each cork-stopper based on the measure of the defects, such as: total area, maximum area, maximum longitudinal crack, maximum transversal crack and top or bottom maximum (border) defects. This classification must only be done if the cork-stopper length belongs to the programmed range. Otherwise, the cork-stopper should be rejected from all classes. The implemented decision software algorithm allows the user to program the claimed values.

In [18] a lot of image features are considered. In this paper there is a strong emphasis on the stability of each feature as they form the basis for any classification procedure. Hence, the focus on the presented five criteria that already allow for good results in classification. A classification rate is not that easy to display because it depends on the cork distribution. A cork placed in the middle of a class can achieve a rate of $100 \%$ while corks that sit on the boundaries can have lower rates. The final classification rate also depends on the number of quality groups where corks must be imputed.

"The worst case criterion" is performed. The user inserts a table containing the maximum acceptable values for each class and for each parameter. The resulting values from the processing algorithms are compared to the inserted values from the user. Each parameter chooses a class. The worst classification decides the final class of the cork-stopper.

An example of user configuration is presented in Table 2. Length measures are in millimetres and area measures are in squared millimetres.

Table 2. User configuration example.

\begin{tabular}{|l|c|c|c|c|c|c|}
\hline & $\begin{array}{l}\mathbf{1}^{\text {st }} \\
\text { class }\end{array}$ & $\begin{array}{l}\mathbf{2}^{\text {nd }} \\
\text { class }\end{array}$ & $\begin{array}{l}\mathbf{3}^{\text {rd }} \\
\text { class }\end{array}$ & $\begin{array}{l}\mathbf{4}^{\text {th }} \\
\text { class }\end{array}$ & $\cdots$ & $\begin{array}{l}\mathbf{7}^{\text {th }} \\
\text { class }\end{array}$ \\
\hline $\begin{array}{l}\text { Total } \\
\text { Area }\end{array}$ & 25 & 60 & 130 & 240 & $\ldots$ & 1000 \\
\hline $\begin{array}{l}\text { Max. } \\
\text { Area }\end{array}$ & 18 & 40 & 90 & 140 & $\cdots$ & 400 \\
\hline $\begin{array}{l}\text { Max. } \\
\text { Long. } \\
\text { Crack }\end{array}$ & 5 & 9 & 13 & 16 & $\cdots$ & 20 \\
\hline $\begin{array}{l}\text { Max. } \\
\text { Transv. } \\
\text { Crack }\end{array}$ & 11 & 22 & 30 & 39 & $\cdots$ & 54 \\
\hline $\begin{array}{l}\text { Max. } \\
\text { Border } \\
\text { Area }\end{array}$ & 12 & 20 & 35 & 49 & & 100 \\
\hline
\end{tabular}

Bearing in mind the example given above, if a cork-stopper had the parameters presented in Table 3, the final classification result would be the $8^{\text {th }}$ class (the worst). In this particularly case, the cork-stopper had a fewer defects area but a longitudinal crack of 21 millimetres aggravated classification. This cork-stopper is considered as the worst class because it may cause leakage of liquid (typically wine) through the crack.

Other kinds of methods can be used to perform the final classification. Fuzzy-Neural Network [10] or Genetic algorithms [11] are examples of that.

\section{Table 3. Parameters values given from image processing.}

\begin{tabular}{|l|c|}
\hline Parameters & Values \\
\hline Total Area & 70 \\
\hline Maximum Area & 30 \\
\hline Maximum Longitudinal crack & 21 \\
\hline Maximum Transversal crack & 18 \\
\hline Max Border Area & 22 \\
\hline
\end{tabular}

\section{Real-time requirements}

Cork manufacturers and cork quality supervisors, ask for a stable and fast system. The industrial system requires a speed of 12000 cork-stoppers per hour. It means about $300 \mathrm{~ms}$ for each cork-stopper to be acquired, processed and classified. The developed hardware gives us the guarantee of real-time requirements. The hard real-time requirements such as pixel acquisition, conversion and storage are done with a microcontroller. The 280 pixels are acquired in $280 \mu \mathrm{s}$. That is the time to complete the acquisition of one line. This line needs $1.33 \mathrm{~ms}$ to be sent to the network in UDP packets. So, $1.5 \mathrm{~ms}$ is needed to acquire and send a line to the network and $150 \mathrm{~ms}$ is the total time to conclude the acquisition and communication task. This is the hard real-time system that is totally performed by the microcontroller.

The remaining time is about $180 \mathrm{~ms}$ and must be used to process and classify the cork-stopper image on a soft real-time system. Therefore, the processing system, a real-time OS, like QNX, RTLINUX, VxWorks, or any other could be used, but is unnecessary because the hard real-time requirements are dealt with by the microcontroller. If the processing system could not conclude the task for one image and another one arriving, that would not necessarily be a serious problem because the system stores the cork-stoppers in a buffer before the command to store them in the right bins is given. This buffer gives some freedom to the soft realtime system as shown in Figure 17. Of course, the average time of processing should be lesser than $180 \mathrm{~ms}$. 
In practice, a time of 20 or $30 \mathrm{~ms}$ is enough to perform this processing task in a $1 \mathrm{GHz}$ processor.

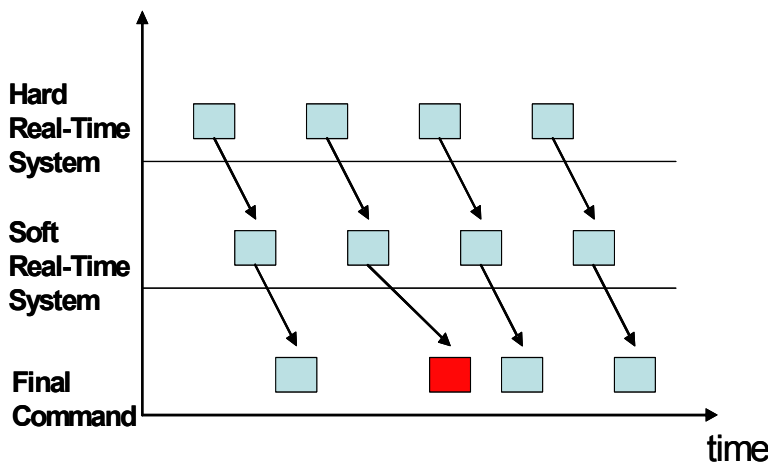

\section{Figure 17. Real-time requirements. Microcontroller controls the Hard Real-Time System and the computer controls the Soft Real-Time System.}

\section{Conclusion}

The developed system acquires and processes images of the cork-stoppers surfaces. The images are acquired through a real-time microcontroller and transmitted through an Ethernet based network. By breaking the system over some almost independent modules, there is an extra degree of freedom in choosing the operating system and development environment. The captured image is processed in a PC so that a qualitative classification for the cork-stopper can be obtained. The quality of the results validates the strategy and algorithms applied.

Another advantage of the decoupled acquisition/processing systems is that it would be easy to use multiple cameras to acquire the images from different angles and furthermore reconfiguration would be an easy task.

As a future work, a coloured camera could be used in order to acquire the RGB components of defects thus improving the selection and classification of a varied type of defects. Some cork-stoppers have a blue speck that does not leak and it will be assumed as a defect by a grey camera which cannot distinguish this blue speck zone from other real defects.

\subsection{References}

[1] M. A. Fortes, "Cork and corks", European Rev., voll, pp. 189-195, 1993.
[2] Atmel Corporation, ATmega128, 2004.

[3] Sony Corporation ILX521A, 1999.

[4] Realtek Corporation, RTL8019AS, 2001.

[5] Kenneth R. Castleman, Digital Image Processing, Prentice Hall, 2000.

[6] Bern Jahne, Digital Image Processing, Concepts, Algorithms and scientific Applications, Springer, 1997.

[7] William K. Pratt, Digital Image Processing, Wiley-Interscience, 1991.

[8] Mark Nixon \& Alberto Aguado, Feature Extraction \& Image Processing, Newnes, 2002.

[9] António Paulo Moreira, Armando Jorge Sousa, Paulo Gomes da Costa, "Vision Based Real-Time Localization Of Multiple Mobile Robots", Proceedings of the 3rd Int. Conf. on Field and Service Robotics, pp.103-106, 2001.

[10] Joongho Chang, Gunhee Han, José M. Valverde, Norman C. Griswold, J. Francisco Duque-Carrillo, Edgar Sánchez-Sinencio, "Cork Quality Classification System using a Unified Image Processing and Fuzzy-Neural Network Methodology", IEEE Transactions on Neural Networks, 1997.

[11] Peech Gourg Nicolas, Hao Jin Kao, "A genetic algorithm for the classification of natural corks", Genetic and Evolutionary Computation Conference, pp. 1382-1388, 2001.

[12] Anacleto Paulo Silva, Américo Azevedo, "Automated Visual Inspection of Cork-Stoppers", Proceedings 11th Portuguese Conference on Pattern Recognition (RECPAD2000), 2000.

[13] Aurélio Campilho, "O reconhecimento de imagens na classificação automática de produtos naturais”, Phd FEUP, 1985.

[14] Tanenbaum Andrew S., Computer networks, Pearson Education International, 2003.

[15] John Fulcher, An introduction to microcomputer systems: architecture and interfacing, Addison-Wesley Publishing Company, 1989.

[16] Triebel, Walter A., The 80386, 80486 and Pentium processors: hardware, software, and interfacing, Prentice-Hall, 1997.

[17] Bruce G. Batchelor, Paul F. Whelan, Intelligent vision systems for industry, Springer, 1997.

[18] Petia Radeva, Marco Bressan, Antonio Tobar, Jordi Vitrià, Real-Time Inspection of Cork Stoppers Using Parametric Methods in High Dimensional Spaces, IASTED International Conference on Signal and Image Processing (SIP 2002), vol. 1, pp. 480-484, USA; 2002.

[19] Natural Cork Quality Council, http://www.corkqc.com. 